\title{
Effects of parenteral nutrition of $\omega-3$ polyunsaturated fatty acid, arginine and glutamine on cellular immune status of patients following liver cancer surgery
}

\author{
Guo Zhixue ${ }^{1}$, Ge Changqing ${ }^{1}$, Han Bing ${ }^{1}$, Qie Zengwang ${ }^{1}$, Kang Yanjie ${ }^{1}$, Liu \\ Bo $^{1}$, Zhu Ya ${ }^{1}$, Wang Weihong ${ }^{1}$, Tian Liqing ${ }^{1}$, Feng Xin ${ }^{1}$, Ma Xiaoyong ${ }^{2 *}$ \\ ${ }^{1}$ Department of Nutriology, The No. 2 Hospital of Baoding, ${ }^{2}$ Department of Orthopeadic Surgery, Affiliated Hospital of Hebei \\ University, Baoding, Hebei 071051, China
}

*For correspondence: Email: pnbcg7@163.com

\begin{abstract}
Purpose: To study the effects of parenteral nutrition (TPN), $\omega-3$ polyunsaturated fatty acid (PUFA), Larginine (Arg), and glutamine on cellular immunity of patients who have done the liver cancer (LC) surgery.

Methods: Seventy-five (75) LC patients were randomly divided into 5 groups (A - E; 15 cases each), group A, B, C, D and E, in which patients were treated with TPN, TPN + fish oil, TPN + Arg, TPN + glutamine, and TPN + $\omega-3$ PUFA + Arg + glutamine, respectively. Before and after surgery, CD3 +, CD4 + and CD8 + were measured by antibody-sensitized erythrocyte rosette test, and IL-6, IL-10 and TNF-a were assayed with double-antibody sandwich enzyme-linked immunoassay (DAS-ELISA). IgA and IgM were measured nephelometrically.

Results: The levels of $C D 3+, C D 4+$ and $C D 8+$ in group $A$ showed no obvious change after surgery ( $p$ $>0.05)$. However, $C D 3+$ and $C D 4+$ increased in groups $B, C$ and $D$, while $C D 8+$ decreased in group $E(p<0.05)$. IL-6 in group $E$ was lower than that in any of the other four groups $(p<0.05)$. IL-10 in group $A$ was lower than that in groups $B, C$ and $D$, but lower than in group $E(p<0.05)$. The levels of TNF-a in groups $B$ and $C$ were lower than those in group $A$, but higher than that in group $E(p<0.05)$ but lower than in group $D$. IgA in group $E$ was higher than in the other groups $(p<0.05)$, while IgM level in group $E$ was lower than in groups $A, B$ and $C(p<0.05)$.

Conclusion: Immunosuppressive status and cellular immunity of patients after liver cancer surgery may be improved by a combination therapy of TPN, $\omega-3$ PUFAs, Arg and glutamine.
\end{abstract}

Keywords: Polyunsaturated fatty acid, Arginine, Glutamine, Parenteral nutrition, Hepatoma, Cellular immunity

\footnotetext{
This is an Open Access article that uses a funding model which does not charge readers or their institutions for access and distributed under the terms of the Creative Commons Attribution License (http://creativecommons.org/licenses/by/4.0) and the Budapest Open Access Initiative (http://www.budapestopenaccessinitiative.org/read), which permit unrestricted use, distribution, and reproduction in any medium, provided the original work is properly credited.

Tropical Journal of Pharmaceutical Research is indexed by Science Citation Index (SciSearch), Scopus, International Pharmaceutical Abstract, Chemical Abstracts, Embase, Index Copernicus, EBSCO, African Index Medicus, JournalSeek, Journal Citation Reports/Science Edition, Directory of Open Access Journals (DOAJ), African Journal Online, Bioline International, Open-J-Gate and Pharmacy Abstracts
}

\section{INTRODUCTION}

Patients with malignant tumors often suffer from malnutrition and immune disfunction. Trauma and post-operative stress response further lower immunity, which often result in poor outcomes and failure of operation [1]. Parenteral nutrition (TPN) can improve the nutritional status of 
postoperative patients, but it cannot restore immunity. However, immune nutrition (the addition of immune nutrient preparations to TPN) can provide postoperative patients with adequate nutrition and inflammation elimination while boosting their immune levels [2]. $\omega-3$ fatty acids and glutamine are common immune nutrients.

In this study, $\omega-3$ unsaturated fatty acids, arginine and glutamine were selected as immune preparations, and the influences of which on cellular immune status of patients after liver cancer surgery were also investigated.

\section{EXPERIMENTAL}

\section{General information on subjects}

In this study, 75 liver cancer patients hospitalized from 2014 to 2016, were selected as study subjects, who were diagnosed with liver cancer through CT, serum AFP examination, and liver biopsy, and who were made up of 45 males and 30 females, aged 40 - 68 years (mean age, 45.8 \pm 7.2 years). There were 35,30 and 10 cases of tumors in the left liver, right liver, and the middle lobe of the liver respectively. Inclusion criteria were: absence of metabolic, infectious and inflammatory bowel diseases; normal liver and kidney functions; absence of other malignant tumors; non-usage of steroids and immunosuppressive agents within 1 month prior to the operation, and non-administration of chemotherapy. Additionally, each patient signed a written informed consent to the participation in the study [3]. Patients who did not meet these inclusion criteria were excluded from the study.

\section{Study methods}

The patients in this study were randomly divided into five groups $(A-E)$, with 15 patients in each one. Patients in group $A$ were treated with TPN, while those in group $B, C, D$ and $E$ received TPN + fish oil, TPN + Arg, TPN + glutamine and TPN $+\omega-3$ PUFA + Arg + glutamine respectively. Twenty (20) patients undergoing open cholecystectomy were selected as control group (with normal immune levels). No significant difference was found in age, sex, number of $\mathrm{T}$ cell sub-types, cellular inflammatory factors and immunoglobulin among the 5 experimental groups.

\section{Composition and dosage of TPN}

TPN was administered to patients after surgery through a central venous catheter placed across the subclavian vein during the operation. The total input procedure was 6 days. In accordance with the nutrition calculation rule, the daily supplemental energy was $30 \mathrm{kcal} / \mathrm{kg}$ and the nitrogen source intake was $0.20 \mathrm{~g} / \mathrm{kg} /$ day, which could guarantee patients' the daily normal nutritional requirement. Compounded by a nutritionist, TPN contained $10 \%$ Compound Amino Acid Injection (Wuxi Huarui Company), 20 $\%$ Lipofundin (fat emulsion; Braun, Germany) and intravenous glucose (50, 10 and $5 \%$ ); in addition to daily intake of adequate vitamins and trace elements. The immune nutrition preparations consisted of $\omega-3$ fish oil, Fat Emulsion Injection (Austria Fresenius Kabi Austria $\mathrm{GmbH}$ ), $25 \mathrm{~g}$ L-Arg/day (Shanghai Biochemical Pharmaceutical Factory), and $0.4 \mathrm{~g}$ glutamine/kg/day (Jiangsu Shenhua Pharmaceutical Co, Ltd), all of which were mixed prior to packaging and presentation.

\section{Evaluation of biochemical indices}

Following manufacturer's instructions, T-cell subtypes CD3 +, CD4 +, CD8 + were assayed with antibody sensitized erythrocyte rosette kits; cellular inflammatory factors IL-6 and IL-10 were determined by applying double antibody sandwich (DAS)-ELISA (Shenzhen Jingmei Company). TNF-a was assayed with TNF-a kit (Shanghai Xinyu Company), while $\operatorname{lgA}$ and IgM levels were determined by nephelometric assay by using kits (American Dade Behring Company).

Ethical approval for the human studies was given by Ethical Committee of Baoding Second Hospital (approval no. 20170101 and the experiments followed international guidelines for human studies [4].

\section{Statistical analysis}

The data are expressed in percentage, and differences were analyzed by t-test. Numerical data are presented as mean \pm standard deviation (SD), and were analyzed by Chi square test using SPSS version 18. $P<0.05$ was taken as indicative of statistically significant difference.

\section{RESULTS}

\section{Levels of T-lymphocyte subsets in patients}

The levels of CD3 + and CD4 + in the liver cancer patients were clearly lower than those of the control group, while CD8 + was higher $(p<$ $0.05)$, which indicated that the cellular immunity of patients with pre-operative liver cancer (PLC) was lower than that of the control group (Table $1)$. 
Table 1: T- cell subsets in pre-operative (PLC) patients

\begin{tabular}{lcccc}
\hline Group & N & CD3 + & CD4 + & CD8 + \\
\hline PLC & 75 & $52.29 \pm$ & $32.12 \pm$ & $36.15 \pm$ \\
& & 6.84 & 4.66 & 5.43 \\
Control & 20 & $70.13 \pm$ & $46.59 \pm$ & $27.14 \pm$ \\
& & 9.20 & 5.07 & 3.64 \\
$t$ value & & -11.66 & 13.88 & -7.42 \\
$p$ value & $<0.01$ & $<0.01$ & $<0.01$ \\
\hline Note: Values & of T-lymphocyte & sub-types \\
expressed as mean \pm SD &
\end{tabular}

Levels of T-cell sub-types in patients after surgery

The levels of CD3 +, CD4 + and CD8 + in group A were of no great difference from their corresponding values prior to surgery $(p>0.05)$, but significantly changed after the operation in the other groups $(p<0.05)$, which indicates that TPN and immune nutrient preparation effectively improved patients' immunity. The CD3 + and CD4 + levels in group E were significantly higher than those in group B, C, D (Table 2), showing that the effect by mixing $\omega-3$ unsaturated fatty acids, Arg and glutamine, was better than that by using these components separately.

Table 2: Number of T-cell subs-types in patients postsurgery

\begin{tabular}{lccc}
\hline Group & CD3 + & CD4 + & CD8 + \\
\hline Pre- & $52.29 \pm$ & $32.12 \pm$ & $36.15 \pm$ \\
surgery & 6.84 & 4.66 & 5.43 \\
A & $53.73 \pm$ & $31.91 \pm$ & $35.67 \pm$ \\
& $5.86^{2}$ & 3.94 & 5.18 \\
B & $58.60 \pm$ & $36.89 \pm$ & $30.17 \pm$ \\
& $5.06^{\star \# \&}$ & $3.59^{\star \# \&}$ & $4.42^{* \# \&}$ \\
C & $59.11 \pm$ & $35.62 \pm$ & $30.28 \pm$ \\
& $4.97^{\star \# \&}$ & $4.01^{* \# \&}$ & $3.60^{\star \# \&}$ \\
D & $59.83 \pm$ & $36.02 \pm$ & $31.09^{\star \#} \pm$ \\
& $4.70^{* \# \&}$ & $4.25^{\star \# \&}$ & $2.88^{* \# \&}$ \\
E & $69.82 \pm$ & $43.58 \pm$ & $26.00 \pm$ \\
\hline
\end{tabular}

Note: Values are mean \pm SD. ${ }^{*} p<0.05$ compared with pre-operative group; \# $p<0.05$ compared with group, ${ }^{\&} p<0.05$ compared with group E. A: Patients received TPN. B: Patients received TPN + fish oil. $\mathrm{C}$ : Patients received TPN + Arg. D: Patients received TPN + glutamine. E: Patients received TPN + $\omega-3$ PUFA + Arg + glutamine

\section{Levels of cellular inflammatory factors in patients post-operation}

No significant difference was in the levels of IL-6 in $B, C$, and $D$ relative to group $A(p>0.05)$, but IL-6 in group $\mathrm{E}$ was obviously lower $(p<0.05)$. Higher amounts of IL-10 were seen in group B, C and $\mathrm{D}$ when compared to group $\mathrm{A}(p<0.05)$, the level of which in $E$ group was significantly higher $(p<0.05)$. Besides, significantly lower levels of TNF-a were obtained in group $B$ and $C$ than in group A $(p<0.05)$. The level of TNF-a in group D was significantly higher than that in group $\mathrm{E}$.

Table 3: Cytokine levels of patients after operation

\begin{tabular}{|c|c|c|c|}
\hline Group & IL-6 (pg/L) & IL-10 (pg/L) & $\begin{array}{l}\text { TNF-a } \\
\text { (pg/L) }\end{array}$ \\
\hline $\begin{array}{l}\text { Before } \\
\text { operation }\end{array}$ & $\begin{array}{c}1590.18 \pm \\
266.02\end{array}$ & $\begin{array}{c}1971.92 \pm \\
279.51\end{array}$ & $\begin{array}{c}3558.72 \pm \\
354.11\end{array}$ \\
\hline A & $\begin{array}{c}1593.57 \pm \\
285.43^{\&}\end{array}$ & $\begin{array}{c}1980.47 \pm \\
323.71^{\&}\end{array}$ & $\begin{array}{c}3591.42 \pm \\
436.58^{\&}\end{array}$ \\
\hline B & $\begin{array}{c}1560.25 \pm \\
271.19^{\&}\end{array}$ & $\begin{array}{l}2038.92 \pm \\
355.64^{\star \# \notin}\end{array}$ & $\begin{array}{c}3278.47 \pm \\
441.50^{\star} \#\end{array}$ \\
\hline C & $\begin{array}{c}1574.73 \pm \\
354.63^{\&}\end{array}$ & $\begin{array}{l}2057.13 \pm \\
302.78^{\star \# \&}\end{array}$ & $\begin{array}{c}3206.28 \pm \\
378.61^{\star \#}\end{array}$ \\
\hline D & $\begin{array}{c}1565.24 \pm \\
297.51^{\&}\end{array}$ & $\begin{array}{l}2069.52 \pm \\
314.53^{* \# \&}\end{array}$ & $\begin{array}{c}3415.09 \pm \\
412.84^{\&}\end{array}$ \\
\hline$E$ & $\begin{array}{c}1510.36 \pm \\
277.35^{\star \#} \\
\end{array}$ & $\begin{array}{l}2292.2 \pm \\
386.55^{\star \# \&} \\
\end{array}$ & $\begin{array}{c}3129.60 \pm \\
386.85^{\star \#}\end{array}$ \\
\hline \multicolumn{4}{|c|}{$\begin{array}{l}\text { Note: Values are mean } \pm \text { SD. } p<0.05 \text { compared } \\
\text { with preoperative group; \#p<0.05 compared with } \\
\text { group A; }{ }^{\circledR} p<0.05 \text { compared with group E. A: Patients } \\
\text { received TPN. B: Patients received TPN + fish oil. C: } \\
\text { Patients received TPN + Arg. D: Patients received } \\
\text { TPN + glutamine. E: Patients received TPN + }+\omega-3 \\
\text { PUFA + Arg + glutamine }\end{array}$} \\
\hline
\end{tabular}

\section{Immunoglobulin content of patients after operation}

Group E had significantly higher IgA than the pre-operative group as well as any of the other four groups $(p<0.05$. The IgA content had no significant difference between group $A$ and group $B, C$ and $D(p>0.05)$. The content of $\lg M$ in group $E$ was clearly lower than that in group $A, B$ and $D(p<0.05)$. These results are shown in Table 4.

Table 4: Immunoglobulin levels in the 5 groups after operation

\begin{tabular}{lcc}
\hline Group & $\operatorname{lgA}(\mathbf{g} / \mathbf{L})$ & $\operatorname{lgM}(\mathbf{g} / \mathbf{L})$ \\
\hline Before & $2.25 \pm 0.58$ & $1.40 \pm 0.30$ \\
operation & & \\
A & $2.20 \pm 0.67^{\star}$ & $1.38 \pm 0.44^{\star}$ \\
B & $2.53 \pm 0.73^{\star}$ & $1.23 \pm 0.28^{\star}$ \\
C & $2.44 \pm 0.86^{\star}$ & $1.03 \pm 0.26^{\star \#}$ \\
D & $2.56 \pm 0.82^{\star}$ & $1.24 \pm 0.22^{\star}$ \\
E & $2.94 \pm 0.71^{\text {*\# }}$ & $1.08 \pm 0.23^{\text {*\# }}$ \\
\hline
\end{tabular}

Note: Values are mean \pm SD; $p<0.05$ in comparison with pre-operative group; ${ }^{\#} p<0.05$ compared with group $A ;{ }^{\&} p<0.05$ in comparison with group $E$. A: Patients received TPN; $B$ : Patients received TPN + fish oil; C: Patients received TPN + Arg; D: Patients received TPN + glutamine; $\mathrm{E}$ : Patients received TPN + $\omega-3$ PUFA + Arg + glutamine

\section{DISCUSSION}

The normal immune function of the human body is maintained by cellular immunity and humoral immunity. The immune system of post-operative 
patients with malignant tumor can be easily affected by such factors as surgical trauma, drugs and malnutrition, although the suppressed immune function is gradually restored to normal 1 month after surgery [5]. What's the problem is that the reduced immunity affects the healing of surgical wounds, may causing post-operative infection and complications. Thus, it is of crucial to provide adequate immune and nutritional support for patients during the post-operative stage, owing to the fact that nutritional support is conducive to patients' recovery, status improvement and further treatment. Studies have demonstrated that T-lymphocyte sub-types and serum cytokines are important indicators of the immune system [6]. This study investigated the effect of added immune nutrients on cellular immunity.

Deep sea fish oil is rich in $\omega-3$ unsaturated fatty acids which can enhance patients' humoral and cellular immune functions, and effectively resist inflammatory reactions. Unsaturated fatty acids as precursors of prostaglandins, are important components of cell membranes, which maintain the fluidity of cell membranes, and participate in the regulation of cellular and biochemical responses [7]. Fish oil/kg body weight/day $(0.2 \mathrm{~g})$ in addition to TPN has been recommended to be taken by gastrointestinal tumor patients after surgery, for reduction of inflammatory response syndrome and post-operative recovery, on the basis of the fact that $\omega-3$ unsaturated fatty acids inhibit the formation of pro-inflammatory factors (IL-6 and TNF-a), increase the synthesis of antiinflammatory factor IL-10, improve immune function and eliminate excessive inflammation $[8,9]$.

As a major component of the mucosal defense system and an important protective barrier, IgA slows down viral proliferation. Appearing in the first humoral immune response, IgM is a marker of infection. T-lymphocytes are the most important immune cells in the human body; CD3 + represents mature $\mathrm{T}$ cells and the overall immune level, while CD4 + stimulates Tlymphocytes directly involved in the immune process, enhancing immunity. On the other hand, CD8 + is a scavenger in the immune process: which removes infected cells, and of which the main role is to suppress immunity. These three immune cells oppose one another, and together balance the immune system [10].

Studies have shown that glutamine supplementation in tumor patients after operation can inhibit the expression of TH-related cytokines, decrease endotoxin translocation and the incidence of post-operative infection, increase glutathione synthesis, and shorten recovery period [11]. In the current study, glutamine supplementation in TPN significantly increased CD3 + and CD4 + levels, and decreased CD8 + content, compared with their corresponding values before surgery. Participates in the regulation of immunity, Arginine (Arg), an essential amino acid and a source of citrulline and nitric oxide in the body, promotes the production of II-6 and TNF-a by Tlymphocytes. Nitric oxide is a messenger molecule involved in mediating many immune responses [12,13].

Studies have also shown that animals infected with bacteria can improve lymphocyte immune function and survival rate after Arg supplementation [14]. In this study, it was found that addition of Arg to TPN significantly increased the production of anti-inflammatory factor IL10and the synthesis of CD3 + and CD4 +; but decreased the levels of bacterial killer CD8 + . The levels of CD3 + and CD4 + in the liver cancer patients were significantly lower, but the level of CD8 + was higher, than those of the control group, which clearly indicates that the cellular immune level of the pre-operative liver cancer patients was lower than that of the control one.

The pattern of changes in the levels of antiinflammatory factors, cytokines and immunoglobulins in the five groups relative to the pre-operative group clearly signifies an improvement in cellular immunity of the postoperative liver cancer patients by incorporation of $\omega-3$ PUFA, Arg and glutamine into TPN.

\section{Limitations of the study}

Few participants were included in this research. What's more, this is a single-center study. Therefore, caution should be exercised in generalizing the findings.

\section{CONCLUSION}

The findings of this investigation show that the combined use of TPN, $\omega-3$ polyunsaturated fatty acids, arginine and glutamine is more effective than the individual compounds in improving cellular immunity levels and reducing inflammatory responses in postoperative liver cancer patients.

\section{DECLARATIONS}

\section{Conflict of Interest}

No conflict of interest associated with this work. 


\section{Contribution of Authors}

We declare that this work was done by named in this article and all liabilities pertaining to claims relating to the content of this article will be borne by the authors. All authors read and approved the manuscript for publication. Ma Xiaoyong conceived and designed the study, Ge Changqing, Han Bing, Qie Zengwang, Kang Yanjie, Liu Bo, Zhu Ya, Wang Weihong, Tian Liqing, Feng Xin collected and analysed the data, Guo Zhixue wrote the manuscript.

\section{REFERENCES}

1. Zhu MW, Tang DN, Hou J, Wei JM, Hua B, Sun JH, Cui HY. Impact of fish oil enriched total parenteral nutrition on elderly patients after colorectal cancer surgery. Chin Med J 2012; 125(2): 178-180.

2. Shen Hao GM, Tang Weiyong. Effect of glutamine fortified parenteral nutrition on postoperative nutrition and immune function in elderly patients with gastrointestinal malignancies. World's Clin Drug 2014; 35(9): 542-546.

3. Xu Yang XJ, Fang Lihua. Effect of parenteral nutrition added with arginine and glutamine on immune status in patients with hepatocellular carcinoma after operation. Chin J Biochem Pharm 2011; 32(1): 63-65.

4. Organization. WH. Declaration of Helsinki. Br Med $J$ 1996; 313(7070): 1448-1449.

5. Zhao Jianjun BX, Huang Zhen. A prospective comparative study of different nutritional support treatments on the recovery of postoperative liver cancer. J Med Res 2014; 43(4): 75-79.

6. Muller AJ, Scherle PA. Targeting the mechanisms of tumoral immune tolerance with small-molecule inhibitors. Nat Rev Cancer 2006; 6(8): 613-625.
7. Liu Ligang LW, Yao Pengfei. Analysis of meta on postoperative short-term efficacy of $\omega-3$ unsaturated fatty acid emulsion in parenteral nutrition in gastrointestinal malignancy. Chin foundation Clin J General Surgery Department 2015; 22(11): 1338-1346.

8. Wei Z, Wang W, Chen J, Yang D, Yan R, Cai Q. A prospective, randomized, controlled study of $\omega-3$ fish oil fat emulsion-based parenteral nutrition for patients following surgical resection of gastric tumors. Nutr $J$ 2014; 13(1): 25-28.

9. Chen B, Zhou Y, Yang $P$, Wan HW, Wu XT. Safety and efficacy of fish oil-enriched parenteral nutrition regimen on postoperative patients undergoing major abdominal surgery: a meta-analysis of randomized controlled trials. J Parenter Enteral Nutr 2010; 34(4): 387-394.

10. Jiang JW, Ren ZG, Chen LY, Jiang L, Xie HY, Zhou L, Zheng SS. Enteral supplementation with glycylglutamine improves intestinal barrier function after liver transplantation in rats. Int $J$ hepatobiliary and pancreatic dis 2011; 10(04): 380-385.

11. Fan YP, Yu JC, Kang WM, Zhang Q. Effects of Glutamine Supplementation on Patients Undergoing Abdominal Surgery. Chin Med Sci J 2009; 24(1): 55-57.

12. Tsuei BJ, Bernard AC, Barksdale AR, Rockich AK, Meier $C F$, Kearney PA. Supplemental enteral arginine is metabolized to ornithine in injured patients. J Surg Res 2005; 123(1): 17-19.

13. Yeh CL, Lee CH, Chen SC, Hou YC, Yeh SL. Effects of arginine-containing total parenteral nutrition on $N$ balance and phagocytic activity in rats undergoing a partial gastrectomy. Br J Nutr 2005; 93(2): 267-272.

14. Daniel TJ, Alexander M, Hubbard WJ, Chaudry $I H$, Choudhry MA, Schwacha MG. Nitric oxide contributes to the development of a post-injury Th2 T-cell phenotype and immune dysfunction. J Cell Physiol 2006; 208(2): 418-420. 